\title{
INFEZIONE DA TOXOCARA SPP. DESCRIZIONE DI UN CASO CLINICO
}

Piccoli S., Grisolia V., Covino F., Rossano F.

Azienda Università Policlinico,

Università degli Studi di Napoli Federico II

Area Funzionale di Diagnostica Microbiologica

La migrazione di larve di Toxocara canis o Toxocara cati nell'uomo è responsabile di manifestazioni cliniche conosciute col nome di sindromi da Larva Migrans Viscerale (LMV), Oculare (LMO), Encefalica (LME).

L'infezione è cosmopolita, colpisce persone che vivono in 
ambienti frequentati da cani e/o gatti come allevatori e veterinari; attualmente è inquadrata come una zoonosi emergente in ambito urbano a causa dell'incontrollata contaminazione del verde pubblico con escrementi canini; per tale motivo la malattia colpisce in modo sempre più allarmante $\mathrm{i}$ bambini, che, portando alla bocca oggetti o mani sporche, si contaminano con le uova presenti nel terreno. È infatti in questo ambiente caldo umido che esse maturando divengono infettanti e, una volta ingerite, liberano le larve che passano attraverso la parete intestinale, giungono al circolo portale e da qui si distribuiscono in tutto l'organismo, soprattutto occhio e s.n.c.

Nell'uomo non è possibile la ricerca diretta delle uova nelle feci, non essendovi un ciclo enterofecale, l'unica possibilità di fare diagnosi, è la ricerca degli anticorpi IgG prodotti verso gli antigeni escretori-secretori $(\mathrm{E} / \mathrm{S})$ generati dalle larve di Toxocara. Il dosaggio di questi, con metodo ELISA, in uso presso il nostro laboratorio, ha dato risultati estremamente soddisfacenti. È questo il caso di un bambino di cinque anni che da mesi presentava febbricola, orticaria e saltuariamente crisi simil epilettiche.

Il quadro radiologico, l'EEG e la TAC escludevano lesioni focali in sede cerebrale.

Gli esami di routine erano normali, ad eccezione di una persistente e inspiegabile ipereosinofilia (65\%).

In seguito a nostra consulenza parassitologica venivano praticate alcune indagini sierologiche tra cui la determinazione di anticorpi anti Toxocara, che dava risultato positivo e che si riconfermava in successivi controlli.

Il bambino veniva sottoposto a terapia con tiabendazolo e, nei mesi successivi, si evidenziarono una diminuzione del titolo anticorpale e la scomparsa dei sintomi clinici e dell'eosinofilia. 